\title{
Toward Deconfounding the Influence of Entity Demographics for Question Answering Accuracy
}

\author{
Maharshi Gor* \\ CS \\ University of Maryland \\ mgoracs. umd. edu
}

\author{
Kellie Webster \\ Google Research, New York \\ websterkegoogle.com
}

\author{
Jordan Boyd-Graber* \\ CS, UMIACS, iSchool, LCS \\ University of Maryland \\ jbg@umiacs.umd.edu
}

\begin{abstract}
The goal of question answering (QA) is to answer any question. However, major QA datasets have skewed distributions over gender, profession, and nationality. Despite that skew, model accuracy analysis reveals little evidence that accuracy is lower for people based on gender or nationality; instead, there is more variation on professions (question topic). But QA's lack of representation could itself hide evidence of bias, necessitating QA datasets that better represent global diversity.
\end{abstract}

\section{Introduction}

Question answering (QA) systems have impressive recent victories-beating trivia masters (Ferrucci et al., 2010) and superhuman reading (Najberg, 2018) - but these triumphs hold only if they generalize; QA systems should be able to answer questions even if they do not look like training examples. While other work (Section 4) focuses on demographic representation in NLP resources, our focus is how well QA models generalize across demographic subsets.

After mapping mentions to a knowledge base (Section 2), we show existing QA datasets lack diversity in the gender and national origin of the people mentioned: English-language QA datasets mostly ask about US men from a few professions (Section 2.2). This is problematic because most English speakers (and users of English QA systems) are not from the US or UK. Moreover, multilingual QA datasets are often translated from English datasets (Lewis et al., 2020; Artetxe et al., 2019). However, no work has verified that QA systems generalize to infrequent demographic groups.

Section 3 investigates whether statistical tests reveal patterns on demographic subgroups. Despite skewed distributions, accuracy is not correlated with gender or nationality, though it is with

\footnotetext{
* Work completed while at Google Research
}

\begin{tabular}{|c|c|c|c|c|c|c|c|c|}
\hline \multirow{2}{*}{ Entity Type } & \multicolumn{2}{|c|}{ NQ } & \multicolumn{2}{|c|}{ QB } & \multicolumn{2}{|c|}{ SQUAD } & \multicolumn{2}{|c|}{ TriviaQA } \\
\hline & Train & Dev & Train & Dev & Train & Dev & Train & Dev \\
\hline Person & 32.14 & 32.27 & 58.49 & 57.18 & 14.56 & 9.56 & 40.89 & 40.69 \\
\hline No entity & 21.97 & 22.61 & 15.88 & 20.13 & 37.27 & 47.95 & 14.85 & 14.93 \\
\hline Location & 28.42 & 27.82 & 34.50 & 35.11 & 33.52 & 29.34 & 44.32 & 44.10 \\
\hline Work of art & 17.31 & 16.19 & 27.98 & 28.16 & 3.15 & 1.15 & 17.53 & 17.76 \\
\hline Other & 2.66 & 2.70 & 9.83 & 9.88 & 5.08 & 3.10 & 14.38 & 14.61 \\
\hline Organization & 17.73 & 18.05 & 20.37 & 17.78 & 21.34 & 19.14 & 22.15 & 21.14 \\
\hline Event & 8.15 & 8.89 & 8.87 & 8.75 & 3.16 & 3.01 & 8.59 & 8.73 \\
\hline Product & 0.85 & 1.06 & 0.88 & 0.63 & 1.39 & 0.33 & 3.99 & 3.99 \\
\hline Total Examples & 106926 & 2631 & 112927 & 2216 & 130319 & 11873 & 87622 & 11313 \\
\hline
\end{tabular}

Table 1: Coverage (\% of examples) of entity-types in QA datasets. Since examples can mention more than one entity, columns can sum to $>100 \%$. Most datasets except SQuAD are rich in people.

professional field. For instance, Natural Questions (Kwiatkowski et al., 2019, NQ) systems do well with entertainers but poorly with scientists, which are handled well in TriviaQA. However, absence of evidence is not evidence of absence (Section 5), and existing QA datasets are not yet diverse enough to vet QA's generalization.

\section{Mapping Questions to Entities}

We analyze four QA tasks: NQ, ${ }^{1}$ SQuAD (Rajpurkar et al., 2016), QB (Boyd-Graber et al., 2012) and TriviaQA (Joshi et al., 2017). Google CLOUD-NL ${ }^{2}$ finds and links entity mentions in QA examples. ${ }^{3}$

\subsection{Focus on People}

Many entities appear in examples (Table 1) but people form a majority in our QA tasks (except SQuAD). Existing work in AI fairness focuses on disparate impacts on people, and models harm especially when it comes to people; hence, our primary intent is to understand how demographic characteristics of "people" correlate with model correctness.

The people asked about in a question can be in the answer- "who founded Sikhism?" (A: Guru

\footnotetext{
${ }^{1}$ For NQ, we only consider questions with short answers.

$2_{\text {https://cloud.google.com/natural-language/docs/ }}$ analyzing-entities

${ }^{3}$ We analyze the dev fold, which is consistent with the training fold (Table 1 and 2), as we examine accuracy.
} 
Nanak), in the question- "what did Clara Barton found?" (A: American Red Cross), or the title of the source document- "what play featuring General Uzi premiered in Lagos in 2001?" (A: King Baabu is in the page on Wole Soyinka). We search until we find an entity: first in the answer, then the question if no entity is found in the answer, and finally the document title.

Demographics are a natural way to categorize these entities and we consider the high-coverage demographic characteristics from Wikidata. ${ }^{4}$ Given an entity, Wikidata has good coverage for all datasets: gender (>99\%), nationality $(>93 \%)$, and profession ( $>94 \%)$. For each characteristic, we use the knowledge base to extract the specific value for a person (e.g., the value "poet" for the characteristic "profession"). However, the values defined by Wikidata have inconsistent granularity, so we collapse near-equivalent values (E.g., "writer", "author", "poet", etc. See Appendix A.1A.2 for an exhaustive list). For questions with multiple values (where multiple entities appear in the answer, or a single entity has multiple values), we create a new value concatenating them together. An 'others' value subsumes values with fewer than fifteen examples; people without a value become 'not found' for that characteristic.

Three authors manually verify entity assignments by vetting fifty random questions from each dataset. Questions with at least one entity had near-perfect $96 \%$ inter-annotator agreement for CLOUD-NL's annotations, while for questions where CLOUD-NL didn't find any entity, agreement is $98 \%$. Some errors were benign: incorrect entities sometimes retain correct demographic values; e.g., Elizabeth II instead of Elizabeth I. Other times, coarse-grained nationality ignores nuance, such as the distinction between Greece and Ancient Greece.

\subsection{Who is in Questions?}

Our demographic analysis reveals skews in all datasets, reflecting differences in task focus (Table 2). NQ are search queries and skew toward popular culture. QB nominally reflects an undergraduate curriculum and captures more "academic" knowledge. TriviaQA is popular trivia, and SQuAD reflects Wikipedia articles.

Across all datasets, men are asked about more than women, and the US is the subject of the majority of questions except in TriviaQA, where the

\footnotetext{
$4_{\text {https://www.wikidata.org/wiki/Wikidata:Database_download }}$
}

\begin{tabular}{|c|c|c|c|c|c|c|c|c|c|}
\hline & \multirow{2}{*}{ Value } & \multicolumn{2}{|c|}{$\mathrm{NQ}$} & \multicolumn{2}{|c|}{ QB } & \multicolumn{2}{|c|}{ SQUAD } & \multicolumn{2}{|c|}{ TriviaQA } \\
\hline & & Train & Dev & Train & Dev & Train & Dev & Train & Dev \\
\hline \multirow{3}{*}{ 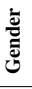 } & Male & 75.67 & 76.33 & 91.77 & 91.63 & 87.82 & 95.15 & 83.76 & 83.32 \\
\hline & Female & 27.47 & 27.56 & 10.29 & 9.87 & 13.44 & 5.20 & 20.54 & 20.29 \\
\hline & No Gender & 0.31 & 0.47 & 0.35 & 0.39 & 0.27 & 0.00 & 0.30 & 0.35 \\
\hline \multirow{6}{*}{ ह } & US & 59.62 & 58.66 & 29.70 & 26.28 & 32.74 & 24.93 & 31.32 & 30.91 \\
\hline & UK & 15.76 & 15.78 & 17.92 & 17.68 & 19.66 & 16.83 & 41.92 & 41.32 \\
\hline & France & 1.79 & 1.18 & 10.06 & 10.34 & 7.76 & 10.57 & 4.37 & 4.84 \\
\hline & Italy & 1.83 & 1.88 & 8.07 & 10.50 & 9.00 & 3.88 & 3.75 & 3.48 \\
\hline & Germany & 1.52 & 2.12 & 7.21 & 6.71 & 4.77 & 6.61 & 3.01 & 3.00 \\
\hline & No country & 4.82 & 4.36 & 7.12 & 6.79 & 3.48 & 2.56 & 6.19 & 6.10 \\
\hline \multirow{8}{*}{ 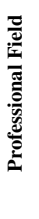 } & Film/TV & 39.19 & 37.93 & 3.16 & 1.89 & 10.72 & 1.32 & 20.64 & 20.75 \\
\hline & Writing & 7.40 & 6.95 & 36.62 & 36.39 & 10.46 & 6.70 & 18.41 & 18.05 \\
\hline & Politics & 11.98 & 10.84 & 24.02 & 24.86 & 36.97 & 46.61 & 21.18 & 20.72 \\
\hline & Science/Tech & 3.61 & 4.71 & 8.93 & 7.50 & 13.67 & 29.60 & 5.43 & 5.54 \\
\hline & Music & 24.08 & 23.44 & 9.51 & 10.73 & 13.56 & 2.64 & 16.46 & 17.38 \\
\hline & Sports & 13.69 & 16.49 & 1.31 & 0.32 & 2.99 & 1.76 & 11.03 & 11.19 \\
\hline & Stage & 15.67 & 14.84 & 1.49 & 1.03 & 1.21 & 1.23 & 10.89 & 10.28 \\
\hline & Artist & 1.84 & 2.47 & 9.17 & 9.00 & 4.58 & 2.38 & 5.75 & 5.93 \\
\hline
\end{tabular}

Table 2: Coverage ( $\%$ of examples) of demographic values across examples with people in QA datasets. Men dominate, as do Americans.

plurality of questions are about the UK. NQ has the highest coverage of women through its focus on entertainment (Film/TV, Music and Sports).

\section{What Questions can QA Answer?}

QA datasets have different representations of demographic characteristics; is this focus benign or do these differences carry through to model accuracy?

We analyze a SOTA system for each of our four tasks. For NQ and SQuAD, we use a fine-tuned BERT (Alberti et al., 2019) with curated training data (e.g., downsample questions without answers and split documents into multiple training instances). For the open-domain TriviaQA task, we use ORQA (Lee et al., 2019) with a BERT-based reader and retriever components. Finally, for QB, we use the competition winner from Wallace et al. (2019), a BERT-based reranker of a TF-IDF retriever. Accuracy (exact-match) and average F1 are both common QA metrics (Rajpurkar et al., 2016). Since both are related and some statistical tests require binary scores, we focus on exact-match.

Rather than focus on aggregate accuracy, we focus on demographic subsets' accuracy (Figure 1). For instance, while $66.2 \%$ of questions about people are correct in $\mathrm{QB}$, the number is lower for the Dutch (Netherlands) (55.6\%) and higher for Ireland $(87.5 \%)$. Unsurprisingly, accuracy is consistently low on the 'not_found' subset, where Wikidata lacks a person's demographic value.

Are the differences we observe across strata significant? We probe this in two ways: using $\chi^{2}$ tests (Plackett, 1983) to see if trends exist and using logistic regression to explore those that do. 

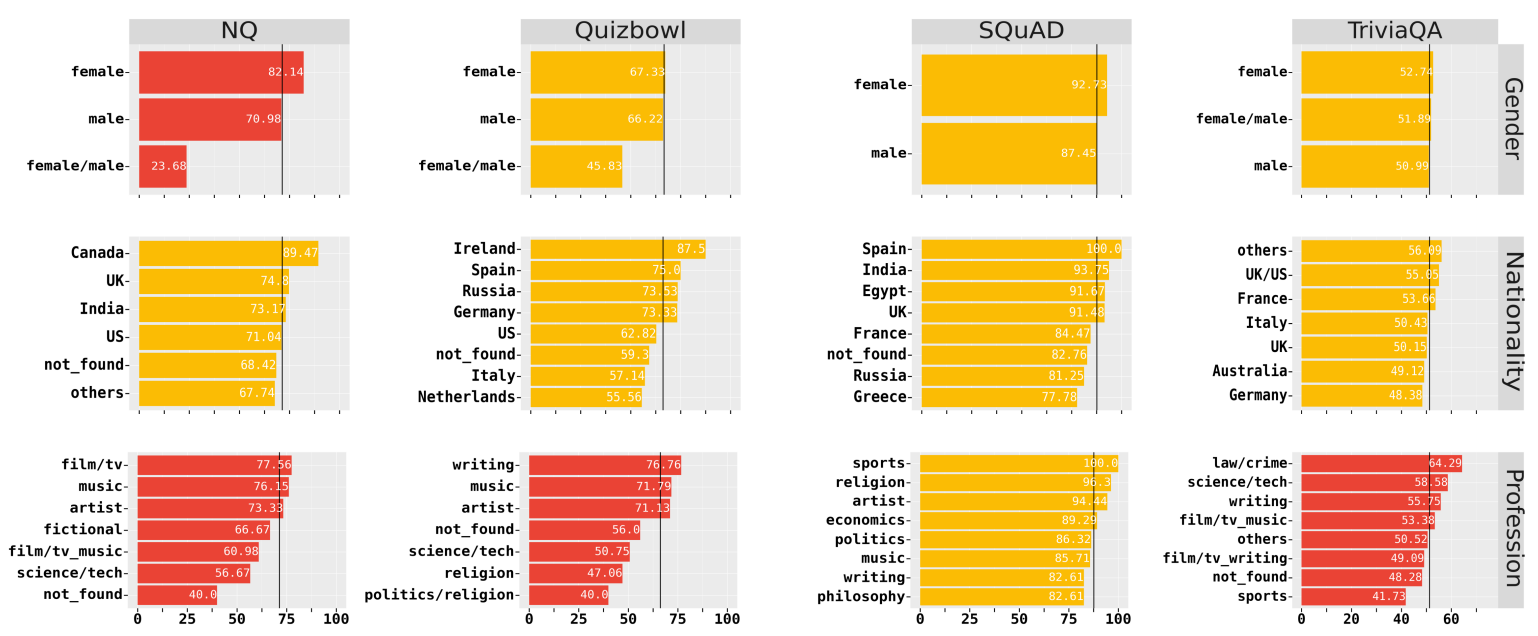

Figure 1: Accuracies split by demographic subsets in our QA datasets' dev fold for all three characteristics compared to average accuracy (vertical line). For each dataset, we only consider examples that have a mention of a person-entity in either the answer, question or document title. Individual plots correspond to a $\chi^{2}$ test on whether demographic values and accuracy are independent (Section 3.1) with the significant characteristics highlighted in red $(p$-value $<0.0167)$.

\subsection{Do Demographic Values Affect Accuracy?}

The $\chi^{2}$ test is a non-parametric test of whether two variables are independent. To see if accuracy and characteristics are independent, we apply a $\chi^{2}$ test to a $n \times 2$ contingency table with $n$ rows representing the frequency of that characteristic's subsets contingent on whether the model prediction is correct or not (Table 3). If we reject the null with a Bonferroni correction (Holm, 1979, divide the $p$-value threshold by three, as we have multiple tests for each dataset), that suggests possible relationships: gender in $\mathrm{NQ}\left(p=2.36 \times 10^{-12}\right)$ and professional field in NQ $(p=0.0142)$, QB $\left(p=2.34 \times 10^{-7}\right)$ and TriviaQA $(p=0.0092)$. However, we find no significant relationship between nationality and accuracy in any dataset.

While $\chi^{2}$ identifies which characteristics impact model accuracy, it does not characterize how. For instance, $\chi^{2}$ indicates NQ's gender is significant, but is this because accuracy is higher for women, or because the presence of both genders in examples lowers the accuracy?

\subsection{Exploration with Logistic Regression}

Thus, we formulate a simple logistic regression: can an example's demographic values predict if a model answers correctly? Logistic regression and related models are the workhorse for discovering and explaining the relationship between variables in history (McCloskey and McCloskey, 1987), ed-

\begin{tabular}{lccc} 
Gender & \# Correct & \# Incorrect & Accuracy \\
\hline male & 433 & 177 & $70.98 \%$ \\
female & 161 & 35 & $82.14 \%$ \\
female/male & 9 & 29 & $23.68 \%$ \\
\hline
\end{tabular}

Table 3: Illustration of $n \times 2$ contingency table for the $\chi^{2}$-test: Gender in $\mathbf{N Q}$, with $n=3$ values: male, female and female/male. Female entities have a higher accuracy $(82 \%)$ than male $(71 \%)$. With two degrees of freedom and $\chi^{2}=53.55$, we get $p=2.4 \times 10^{-12}$, signaling significance.

ucation (van der Linden and Hambleton, 2013), political science (Poole and Rosenthal, 2011), and sports (Glickman and Jones, 1999). Logistic regression is also a common tool in NLP: to find linguistic constructs that allow determiner omission (Kiss et al., 2010) or to understand how a scientific paper's attributes effect citations (Yogatama et al., 2011). Unlike model calibration (Niculescu-Mizil and Caruana, 2005), whose goal it to maximize prediction accuracy, the goal here is explanation.

We define binary features for demographic values of characteristics the $\chi^{2}$ test found significant (thus we exclude all of SQuAD, the nationality characteristic, and gender characteristic for all but NQ). For instance, a question about Abidali Neemuchwala would have features for g_male, o_executive but zero for everything else. ${ }^{5}$ Real-valued features, multi_entities and multi_answers, capture the effect of multiple

\footnotetext{
${ }^{5}$ Exhaustive list of demographic features in the Appendix.
} 
person-entities and multiple gold-answers (scaled with the base two logarithm).

But that is not the only reason an answer may be difficult or easy. Following Sugawara et al. (2018), we incorporate features that reveal the questions' difficulty. For instance, questions that clearly hint the answer type reduce ambiguity. The $t_{-}$who checks if the token "who" is in the start of the question. Similarly, $t \_w h a t, t \_w h e n$, and $t \_w h e r e$ capture other entity-types. Questions are also easier if evidence only differs from the question by a couple of words; thus, q_sim is the Jaccard similarity between question and evidence tokens. Finally, the binary feature e_train_count marks if the personentities occur in the training data more than twice.

We first drop features with negligible effect on accuracy using LASSO (regularization $\lambda=1$ ) by removing zero coefficients. For the remaining features, Wald statistics (Fahrmeir et al., 2007) estimate $p$-values. Although we initially use quadratic features they are all eliminated during feature reduction. Thus, we only report the linear features with a minimal significance $(p$-value $<0.1)$.

\subsection{How do Properties Affect Accuracy?}

Recall that logistic regression uses features to predict whether the QA system will get the answer right or not. Features associated with correct answers have positive weights (like those derived from Sugawara et al. (2018), q_sim and e_train_count), those associated with incorrect answers have negative weights, and features without effect will be near zero. Among the $t_{-} w h *$ features, $t \_w h o$ significantly correlates with model correctness, especially in NQ and QB, where questions asked directly about a person.

However, our goal is to see if, after accounting for obvious reasons a question could be easy, demographic properties can explain QA accuracy. The strongest effect is for professions (Table 4). For instance, while NQ and QB systems struggle on science questions, TriviaQA's does not. Science has roughly equivalent representation (Table 2), suggesting QB questions are harder.

While multi_answer (and multi_entities) reveal harder NQ questions, it has a positive effect in TriviaQA, as TriviaQA uses multiple answers for alternate formulations of answers (Appendix B.2.1, B.2.2), which aids machine reading, while multiple NQ answers are often a sign of ambiguity (BoydGraber and Börschinger, 2020; Si et al., 2021):
"Who says that which we call a rose?" A: Juliet, A: William Shakespeare. For male and female genders, NQ has no statistically significant effect on accuracy, only questions about entities with multiple genders depresses accuracy. Given the many findings of gender bias in NLU (Zhao et al., 2017; Webster et al., 2018; Zhao et al., 2018; Stanovsky et al., 2019), this is surprising. However, we caution against accepting this conclusion without further investigation given the strong correlation of gender with professional field (Goulden et al., 2011), where we do see significant effects.

Taken together, the $\chi^{2}$ and logistic regression analysis give us reason to be optimistic: although data are skewed for all subsets, QA systems might well generalize from limited training data across gender and nationality.

\section{Related Work}

Language is a reflection of culture. Like other cultural artifacts-encyclopedias (Reagle and Rhue, 2011), and films (Sap et al., 2017)—QA has more men than women. Other artifacts like children's books have more gender balance but reflect other aspects of culture (Larrick, 1965).

The NLP literature is also grappling with demographic discrepancies. Standard coreference systems falter on gender-balanced corpora (Webster et al., 2018), and Zhao et al. (2018) create synthetic training data to reduce bias. Similar coreference issues plague machine translation systems (Stanovsky et al., 2019), and Li et al. (2020) use QA to probe biases of NLP systems. Sen and Saffari (2020) show that there are shortcomings in QA datasets and evaluations by analysing their out-of-domain generalization capabilities and ability to handle question variation. Joint models of vision and language suggest that biases come from language, rather than from vision (Ross et al., 2021). However, despite a range of mitigation techniques (Zhao et al., 2017, inter alia) none, to our knowledge, have been successfully applied to QA, especially from the demographic viewpoint.

\section{Discussion and Conclusion}

This paper delivers both good news and bad news. While datasets remain imperfect and reflect societal imperfections, for many demographic properties, we do not find strong evidence that QA suffers from this skew.

However, this is an absence of evidence rather 


\begin{tabular}{|c|c|c|c|c|c|c|}
\hline Dataset & Features & Coef & SE & Wald (|WI) & $\mathbb{P}_{Z \sim \mathcal{N}}(|Z|$ & $>|W|)$ \\
\hline \multirow{4}{*}{ NQ } & bias & +1.964 & 0.727 & 2.703 & 0.0069 & $\star \star \star$ \\
\hline & multi_answers & -1.893 & 0.438 & 4.327 & 0.0000 & 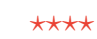 \\
\hline & o_not_found & -1.112 & 0.514 & 2.163 & 0.0305 & $\star \star$ \\
\hline & t_who & +0.773 & 0.280 & 2.764 & 0.0057 & $\star \star \star$ \\
\hline \multirow{4}{*}{$\begin{array}{l}\text { Model Fit: } \\
\mathbf{7 8 . 0 9 \%}\end{array}$} & o_science/tech & -0.715 & 0.390 & 1.832 & 0.0670 & $\star$ \\
\hline & multi_entities & -0.678 & 0.342 & 1.979 & 0.0478 & $\star \star$ \\
\hline & q_sim & +0.406 & 0.210 & 1.934 & 0.0531 & $\star$ \\
\hline & e_train_count & +0.353 & 0.178 & 1.979 & 0.0479 & $\star \star$ \\
\hline \multirow{5}{*}{ QB } & e_train_count & +1.922 & 0.269 & 7.144 & 0.0000 & $x \star x \star x$ \\
\hline & bias & -1.024 & 0.291 & 3.516 & 0.0004 & 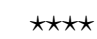 \\
\hline & offilm/tv & -0.910 & 0.470 & 1.934 & 0.0531 & $\star$ \\
\hline & multi_entities & -0.870 & 0.165 & 5.287 & 0.0000 & $\star \star \star \star \star ~$ \\
\hline & o_science/tech & -0.667 & 0.265 & 2.522 & 0.0117 & $\star \star$ \\
\hline \multirow{3}{*}{$\begin{array}{l}\text { Model Fit: } \\
71.90 \%\end{array}$} & o_religion & -0.655 & 0.362 & 1.812 & 0.0700 & $\star$ \\
\hline & o_writing & +0.402 & 0.189 & 2.128 & 0.0334 & $\star \star$ \\
\hline & t_who & +0.363 & 0.183 & 1.990 & 0.0466 & $\star \star$ \\
\hline \multirow{4}{*}{ TriviaQA } & bias & -1.066 & 0.114 & 9.353 & 0.0000 & 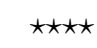 \\
\hline & o_religion & -0.443 & 0.255 & 1.738 & 0.0822 & $\star$ \\
\hline & o_law/crime & +0.412 & 0.218 & 1.890 & 0.0588 & $\star$ \\
\hline & multi_answers & +0.341 & 0.024 & 14.090 & 0.0000 & 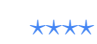 \\
\hline \multirow{3}{*}{$\begin{array}{l}\text { Model Fit: } \\
60.18 \%\end{array}$} & t_who & +0.230 & 0.129 & 1.778 & 0.0754 & $\star$ \\
\hline & o_politics & -0.208 & 0.095 & 2.177 & 0.0295 & $\star \star$ \\
\hline & o_writing & +0.192 & 0.098 & 1.955 & 0.0506 & $\star$ \\
\hline
\end{tabular}

Table 4: Influential features after filtering characteristics based on a $\chi^{2}$ test (Figure 1). Highly influential features ( $p$-value $<0.1$ ), both positive (blue) and negative (red). Higher number of $\star$ 's signals higher significance.

than evidence of absence: these are skewed datasets that have fewer than a quarter of the questions about women. It is difficult to make confident assessments on such small datasets-many demographic values were excluded because they appeared infrequently (or not at all). Improving the diversity of QA datasets can help us be more certain that QA systems do generalize and reflect the diverse human experience. Considering such shortcomings, Rodriguez et al. (2021) advocate improving evaluation by focusing on more important examples for ranking models; demographic properties could further refine more holistic evaluations.

A broader analysis beyond person entities would indeed be a natural extension of this work. Label propagation can expand the analysis beyond people: the Hershey-Chase experiment is associated with Alfred Hershey and Martha Chase, so it would-given the neighboring entities in the Wikipedia link graph-be 100\% American, 50\% male, and 50\% female. Another direction for future work is accuracy under counterfactual perturbation: swapping real-world entities (in contrast with nonce entities in Li et al. (2020)) with different demographic values.

Nonetheless, particularly for professional fields, imbalances remain. The lack of representation in QA could cause us to think that things are better than they are because of Simpson's paradox (Blyth, 1972): gender and profession are not independent! For example, in NQ, our accuracy on women is higher in part because of its tilt toward entertainment, and we cannot say much about women scientists. We therefore caution against interpreting strong model performance on existing QA datasets as evidence that the task is 'solved'. Instead, future work must consider better dataset construction strategies and robustness of accuracy metrics to different subsets of available data, as well as unseen examples.

\section{Acknowledgements}

We thank Michael Collins, Slav Petrov, Tulsee Doshi, Sephora Madjiheurem, Benjamin Börschinger, Pedro Rodriguez, Massimiliano Ciaramita, Kenton Lee, Alex Beutal, Kenton Lee, and Emily Pitler for their early and insightful comments on the proposal and drafts. Additionally, insights about Google's CLOUD-NL entity linking tool and WiKiDATA KB from Jan Botha, Denny Vrandecic, Livio Soares, and Tom Kwiatkowski were useful in designing the entity linking and attribute extraction pipeline. 


\section{Ethical Considerations}

This work analyses demographic subsets across QA datasets based on Gender, Nationality and Profession. We believe the work makes a positive contribution to representation and diversity by pointing out the skewed distribution of existing QA datasets. To avoid noise being interpreted as signal given the lack of diversity in these datasets, we could not include various subgroups that we believe should have been part of this study: non-binary, intersectional groups (e.g., women scientists in $\mathrm{NQ}$ ), people indigenous to subnational regions, etc. We believe increasing representation of all such groups in QA datasets would improve upon the status quo. We infer properties of mentions using Google CloudNL to link the entity in a QA example to an entry in the WIKIDATA knowledge base to attribute gender, profession and nationality. We acknowledge that this is not foolproof and itself vulnerable to bias, although our small-scale accuracy evaluation did not reveal any concerning patterns.

All human annotations are provided by authors to verify entity-linkings and were fairly compensated.

\section{References}

Chris Alberti, Kenton Lee, and Michael Collins. 2019. A BERT baseline for the natural questions. arXiv preprint arXiv:1901.08634.

Mikel Artetxe, Sebastian Ruder, and Dani Yogatama. 2019. On the cross-lingual transferability of monolingual representations. CoRR, abs/1910.11856.

Colin R. Blyth. 1972. On Simpson's paradox and the sure-thing principle. Journal of the American Statistical Association, 67(338):364-366.

Jordan Boyd-Graber and Benjamin Börschinger. 2020. What question answering can learn from trivia nerds. In Proceedings of the Association for Computational Linguistics.

Jordan Boyd-Graber, Brianna Satinoff, He He, and Hal Daume III. 2012. Besting the quiz master: Crowdsourcing incremental classification games. In Proceedings of Empirical Methods in Natural Language Processing.

Ludwig Fahrmeir, Thomas Kneib, Stefan Lang, and Brian Marx. 2007. Regression. Springer.

David Ferrucci, Eric Brown, Jennifer Chu-Carroll, James Fan, David Gondek, Aditya A. Kalyanpur, Adam Lally, J. William Murdock, Eric Nyberg, John Prager, Nico Schlaefer, and Chris Welty. 2010. Building Watson: An Overview of the DeepQA Project. AI Magazine, 31(3).
Mark E Glickman and Albyn C Jones. 1999. Rating the chess rating system. Chance, 12.

Marc Goulden, Mary Ann Mason, and Karie Frasch. 2011. Keeping women in the science pipeline. The Annals of the American Academy of Political and Social Science, 638:141-162.

Sture Holm. 1979. A simple sequentially rejective multiple test procedure. Scandinavian Journal of Statistics, 6(2):65-70.

Mandar Joshi, Eunsol Choi, Daniel Weld, and Luke Zettlemoyer. 2017. TriviaQA: A large scale distantly supervised challenge dataset for reading comprehension. In Proceedings of the Association for Computational Linguistics.

Tibor Kiss, Katja Keßelmeier, Antje Müller, Claudia Roch, Tobias Stadtfeld, and Jan Strunk. 2010. A logistic regression model of determiner omission in PPs. In Proceedings of International Conference on Computational Linguistics, pages 561-569, Beijing, China.

Tom Kwiatkowski, Jennimaria Palomaki, Olivia Redfield, Michael Collins, Ankur Parikh, Chris Alberti, Danielle Epstein, Illia Polosukhin, Matthew Kelcey, Jacob Devlin, Kenton Lee, Kristina N. Toutanova, Llion Jones, Ming-Wei Chang, Andrew Dai, Jakob Uszkoreit, Quoc Le, and Slav Petrov. 2019. Natural questions: A benchmark for question answering research. Transactions of the Association for Computational Linguistics.

Nancy Larrick. 1965. The all-white world of children's books. The Saturday Review, 64:63-65.

Kenton Lee, Ming-Wei Chang, and Kristina Toutanova. 2019. Latent retrieval for weakly supervised open domain question answering. In Proceedings of the Association for Computational Linguistics.

Patrick Lewis, Barlas Oguz, Ruty Rinott, Sebastian Riedel, and Holger Schwenk. 2020. MLQA: Evaluating cross-lingual extractive question answering. In Proceedings of the Association for Computational Linguistics, Online.

Tao Li, Daniel Khashabi, Tushar Khot, Ashish Sabharwal, and Vivek Srikumar. 2020. UNQOVERing stereotyping biases via underspecified questions. In Findings of the Association for Computational Linguistics: EMNLP.

Deirdre N. McCloskey and Donald N. McCloskey. 1987. Econometric History. Casebook Series. Macmillan Education.

Sewon Min, Julian Michael, Hannaneh Hajishirzi, and Luke Zettlemoyer. 2020. AmbigQA: Answering ambiguous open-domain questions. In Proceedings of Empirical Methods in Natural Language Processing, Online.

Adam Najberg. 2018. Alibaba AI model tops humans in reading comprehension. 
Alexandru Niculescu-Mizil and Rich Caruana. 2005 Predicting good probabilities with supervised learning. In Proceedings of the International Conference of Machine Learning.

Robin L Plackett. 1983. Karl Pearson and the chisquared test. International Statistical Review/Revue Internationale de Statistique, pages 59-72.

K.T. Poole and H.L. Rosenthal. 2011. Ideology and Congress. American Studies. Transaction Publishers.

Pranav Rajpurkar, Jian Zhang, Konstantin Lopyrev, and Percy Liang. 2016. SQuAD: 100,000+ questions for machine comprehension of text. In Proceedings of Empirical Methods in Natural Language Processing.

Joseph Reagle and Lauren Rhue. 2011. Gender bias in Wikipedia and Britannica. International Journal of Communication, 5(0).

Pedro Rodriguez, Joe Barrow, Alexander Miserlis Hoyle, John P. Lalor, Robin Jia, and Jordan BoydGraber. 2021. Evaluation examples are not equally informative: How should that change nlp leaderboards? In Proceedings of the Association for Computational Linguistics.

Candace Ross, Boris Katz, and Andrei Barbu. 2021 Measuring social biases in grounded vision and language embeddings. In Conference of the North American Chapter of the Association for Computational Linguistics, Online.

Maarten Sap, Marcella Cindy Prasettio, Ari Holtzman, Hannah Rashkin, and Yejin Choi. 2017. Connotation frames of power and agency in modern films. In Proceedings of Empirical Methods in Natural Language Processing.

Priyanka Sen and Amir Saffari. 2020. What do models learn from question answering datasets? In Proceedings of Empirical Methods in Natural Language Processing.

Chenglei Si, Chen Zhao, and Jordan Boyd-Graber. 2021. What's in a name? answer equivalence for open-domain question answering. In Emperical Methods in Natural Language Processing.

Gabriel Stanovsky, Noah A. Smith, and Luke Zettlemoyer. 2019. Evaluating gender bias in machine translation. In Proceedings of the Association for Computational Linguistics.

Saku Sugawara, Kentaro Inui, Satoshi Sekine, and Akiko Aizawa. 2018. What makes reading comprehension questions easier? In Proceedings of Empirical Methods in Natural Language Processing.

Wim J van der Linden and Ronald K Hambleton. 2013. Handbook of modern item response theory. Springer Science \& Business Media.
Eric Wallace, Pedro Rodriguez, Shi Feng, Ikuya Yamada, and Jordan Boyd-Graber. 2019. Trick me if you can: Human-in-the-loop generation of adversarial question answering examples. Transactions of the Association of Computational Linguistics, 10.

Kellie Webster, Marta Recasens, Vera Axelrod, and Jason Baldridge. 2018. Mind the GAP: A balanced corpus of gendered ambiguous pronouns. Transactions of the Association for Computational Linguistics, 6:605-617.

Dani Yogatama, Michael Heilman, Brendan O'Connor, Chris Dyer, Bryan R. Routledge, and Noah A. Smith. 2011. Predicting a scientific community's response to an article. In Proceedings of Empirical Methods in Natural Language Processing.

Jieyu Zhao, Tianlu Wang, Mark Yatskar, Vicente Ordonez, and Kai-Wei Chang. 2017. Men also like shopping: Reducing gender bias amplification using corpus-level constraints. In Proceedings of Empirical Methods in Natural Language Processing.

Jieyu Zhao, Tianlu Wang, Mark Yatskar, Vicente Ordonez, and Kai-Wei Chang. 2018. Gender bias in coreference resolution: Evaluation and debiasing methods. In Conference of the North American Chapter of the Association for Computational Linguistics. 


\section{Appendix}

\section{A Entity collapses of demographic values}

While mapping QA examples to person entities and values for their corresponding demographic characteristics (Section 2), we encountered many nearby values: 'Poet', 'Writer', 'Author'. We collapse such values into a single label which we use for further analysis. This section enlists all the collapses that we encounter for determining nationality of people (Appendix A.1) and their professions (Appendix A.2).

\section{A.1 Entity-collapses for Nationality values}

US: kingdom of hawaii, united states, united states of america

UK: commonwealth of england, great britain, kingdom of england, kingdom of mercia, kingdom of scotland, kingdom of wessex, united kingdom, united kingdom of great britain and ireland

Albania: kingdom of albania

Austria: austrian empire, federal state of austria, first republic of austria

Cyprus: kingdom of cyprus, republic of cyprus, turkish republic of northern cyprus

Denmark: kingdom of denmark

France: kingdom of france

Germany: german confederation, german democratic republic, german empire, german reich, germany, kingdom of hanover, kingdom of prussia, kingdom of saxony, nazi germany, north german confederation, prussia, republic of german-austria, west germany

Greece: ancient greece, greece
Hungary: hungary, kingdom of hungary, people's republic of hungary

Ireland: irish republic, kingdom of ireland

Italy: ancient rome, florence, holy roman empire, kingdom of italy, kingdom of sardinia

Netherlands: dutch republic, kingdom of the netherlands

Poland: kingdom of poland, poland

Portugal: kingdom of portugal

Romania: kingdom of romania, romania, socialist republic of romania

Spain: crown of castile, kingdom of aragon, kingdom of castile, kingdom of navarre, spain

Yugoslavia: federal republic of yugoslavia, kingdom of yugoslavia, socialist federal republic of yugoslavia, yugoslavia

Iraq: ba'athist iraq, iraq, kingdom of iraq, mandatory iraq, republic of iraq (1958-68)

Israel: israel, kingdom of israel, land of israel

Russia: russia, russian empire, russian soviet federative socialist republic, soviet union, tsardom of russia

India: british raj, delhi sultanate, dominion of india, india 
China: china, people's republic of china, republic of china (1912-1949)

Egypt : ancient egypt, egypt, kingdom of egypt, republic of egypt

\section{A.2 Entity-collapses for Profession values}

Writing: author, biographer, cartoonist, children's writer, comedy writer, comics artist, comics writer, contributing editor, cookery writer, detective writer, diarist, editor, editorial columnist, essayist, fairy tales writer, grammarian, hymnwiter, journalist, lexicographer, librettist, linguist, literary, literary critic, literary editor, literary scholar, memoirist, newspaper editor, non-fiction writer, novelist, opinion journalist, philologist, photojournalist, physician writer, playwright, poet, poet lawyer, preface author, prosaist, religious writer, science fiction writer, science writer, scientific editor, screenwriter, short story writer, tragedy writer, travel writer, women letter writer, writer

Sports: amateur wrestler, american football coach, american football player, archer, artistic gymnast, association football manager, association football player, association football referee, athlete, athletics competitor, australian rules football player, badminton player, ballet dancer, ballet master, ballet pedagogue, baseball player, basketball coach, basketball player, biathlete, biathlon coach, boxer, bridge player, canadian football player, chess player, choreographer, coach, cricket umpire, cricketer, dancer, darts player, field hockey player, figure skater, figure skating choreographer, figure skating coach, formula one driver, gaelic football player, golfer, gridiron football player, gymnast, head coach, hurler, ice dancer, ice hockey coach, ice hockey player, jockey, judoka, lacrosse player, long-distance runner, marathon runner, marimba player, martial artist, middle-distance runner, mixed martial artist, motorcycle racer, poker player, polo player, pool player, professional wrestler, quidditch player, racing automobile driver, racing driver, rink hockey player, rugby league player, rugby player, rugby union coach, rugby union player, runner, short track speed skater, skateboarder, skeleton racer, snooker player, snowboarder, sport cyclist, sport shooter, sporting director, sports agent, sports commentator, sprinter, squash player, surfer, swimmer, table tennis player, taekwondo athlete, tennis coach, tennis player, thai boxer, track and field coach, viol player, volleyball player, water polo player

Music: bass guitar, bassist, blues musician, child singer, classical composer, classical guitarist, classical pianist, collector of folk music, composer, conductor, country musician, drummer, film score composer, ghost singer, guitar maker, guitarist, heavy metal singer, instrument maker, instrumentalist, jazz guitarist, jazz musician, jazz singer, keyboardist, lyricist, multi-instrumentalist, music arranger, music artist, music critic, music director, music interpreter, music pedagogue, music pedagogy, music producer, music publisher, music theorist, music video director, musical, musical instrument maker, musician, musicologist, opera composer, opera singer, optical instrument maker, organist, pianist, playback singer, professor of music composition, rapper, record producer, recording artist, rock drummer, rock musician, saxophonist, session musician, singer, singer-songwriter, songwriter, violinist

Fictional: fictional aviator, fictional businessperson, fictional character, fictional cowboy, fictional domestic worker, fictional firefighter, fictional journalist, fictional mass murderer, fictional pirate, fictional police officer, fictional politician, fictional schoolteacher, fictional scientist, fictional seaman, fictional 
secretary, fictional soldier, fictional space traveller, fictional taxi driver, fictional vigilante, fictional waitperson, fictional writer

Politics: activist, ambassador, animal rights advocate, anti-vaccine activist, civil rights advocate, civil servant, climate activist, colonial administrator, consort, dictator, diplomat, drag queen, duke, emperor, feminist, foreign minister, government agent, governor, human rights activist, internet activist, khan, king, leader, lgbt rights activist, military commander, military leader, military officer, military personnel, military theorist, minister, monarch, peace activist, political activist, political philosopher, political scientist, political theorist, politician, president, prince, princess, protestant reformer, queen, queen consort, queen regnant, religious leader, revolutionary, ruler, secretary, social reformer, socialite, tribal chief

Artist: architect, artist, baker, blacksmith, car designer, chef, costume designer, design, designer, fashion designer, fashion photographer, fresco painter, furniture designer, game designer, glass artist, goldsmith, graffiti artist, graphic artist, graphic designer, house painter, illustrator, industrial designer, interior designer, jewellery designer, landscape architect, landscape painter, lighting designer, painter, photographer, postage stamp designer, printmaker, production designer, scientific illustrator, sculptor, sound designer, textile designer, type designer, typographer, visual artist

Film/tv: actor, character actor, child actor, documentary filmmaker, dub actor, factory owner, fashion model, film actor, film critic, film director, film editor, film producer, filmmaker, glamour model, line producer, model, pornographic actor, reality television participant, runway model, television actor, television director, television editor, television presenter, television producer, voice actor

Executive: bank manager, business executive, business magnate, businessperson, chief executive officer, entrepreneur, executive officer, executive producer, manager, real estate entrepreneur, talent manager

Stage: circus performer, comedian, entertainer, mime artist, musical theatre actor, stage actor, stand-up comedian, theater director

Law/crime: art thief, attorney at law, bank robber, canon law jurist, courtier, criminal, judge, jurist, lawyer, official, private investigator, robber, serial killer, spy, thief, war criminal

History: anthropologist, archaeologist, art historian, church historian, classical archaeologist, egyptologist, explorer, historian, historian of classical antiquity, historian of mathematics, historian of science, historian of the modern age, labor historian, legal historian, military historian, paleoanthropologist, philosophy historian, literary historian, music historian, paleontologist, polar explorer,

Science/tech: aerospace engineer, alchemist, anesthesiologist, artificial intelligence researcher, astrologer, astronaut, astronomer, astrophysicist, auto mechanic, bacteriologist, biochemist, biologist, botanist, bryologist, cardiologist, chemical engineer, chemist, chief engineer, civil engineer, climatologist, cognitive scientist, combat engineer, computer scientist, cosmologist, crystallographer, earth scientist, ecologist, educational psychologist, electrical engineer, engineer, environmental scientist, epidemiologist, 


\begin{abstract}
ethnologist, ethologist, evolutionary biologist, geochemist, geographer, geologist, geophysicist, immunologist, industrial engineer, inventor, marine biologist, mathematician, mechanic, mechanical automaton engineer, mechanical engineer, meteorologist, microbiologist, mining engineer, naturalist, neurologist, neuroscientist, nuclear physicist, nurse, ontologist, ornithologist, patent inventor, pharmacologist, physician, physicist, physiologist, planetary scientist, psychiatrist, psychoanalyst, psychologist, railroad engineer, railway engineer, research assistant, researcher, scientist, social psychologist, social scientist, sociologist, software engineer, space scientist, statistician, structural engineer, theoretical biologist, theoretical physicist, virologist, zoologist
\end{abstract}

Education: academic, adjunct professor, associate professor, educator, head teacher, high school teacher, history teacher, lady margaret's professor of divinity, pedagogue, professor, school teacher, sex educator, teacher, university teacher

Economics: economist

Religion: anglican priest, bible translator, bishop, catholic priest, christian monk, lay theologian, monk, pastor, pope, preacher, priest, theologian

Military: air force officer, aircraft pilot, commanding officer, fighter pilot, general officer, helicopter pilot, intelligence officer, naval officer, officer of the french navy, police officer, soldier, starship pilot, test pilot

Translation: translator

Philosophy: analytic philosopher, philosopher, philosopher of language, philosopher of science

Polymath: polymath 


\section{B Logistic Regression features.}

This section enlists a full set of features used for the logistic regression analysis after feature reduction, each with their coefficients, standard error, Wald Statistic and significance level in Table 5. We also describe the templates and the implementation details of the features using in our logistic regression analysis (Section 3.2) in Appendix B.1, and finally enlist some randomly sampled examples both from NQ and TriviaQA datasets in Appendix B.2 to show how multi_answers feature has disparate effects on them.

\section{B.1 Implementation of Logistic Regression features}

- q_sim: For closed-domain QA tasks like NQ and SQuAD, this feature measures (sim)ilarity between (q)uestion text and evidence sentence- the sentence from the evidence passage which contains the answer text—using Jaccard similarity over unigram tokens (Sugawara et al., 2018). Since we do not include SQuAD in our logistic regression analysis (Section 3.2, this feature is only relevant for NQ.

- e_train_count: This binary feature represents if distinct (e)ntities appearing in a QA example (through the approach described in Section 2) appears more than twice in the particular dataset's training fold. We avoid logarithm here as even the log frequency for some commonly occurring entities exceeds the expected feature value range.

- $t \_w h *$ : This represents the features that captures the expected entity type of the answer: t_who, t_what, t_where, t_when. Each binary feature captures if the particular "wh*" word appears in the first ten (t)okens of the question text. $^{6}$

- multi_entities: For number of linked person-entities in a example as described in Section 2 as $n$, this feature is $\log _{2}(n)$. Hence, this feature is 0 for example with just single person entity.

- multi_answers: For number of gold-answers annotated in a example as $n$, this feature is

\footnotetext{
${ }^{6} \mathrm{QB}$ questions often start with "For 10 points, name this writer who..."
}

$\log _{2}(n)$. Hence, this feature is 0 for example with just answer.

- $g_{-} \star$ : Binary demographic feature signaling the presence of the (g)ender characterized by the feature. For instance, g_female signals if the question is about a female person.

- ○_*: Binary demographic feature signaling the presence of the occupation (or profession) as characterized by the feature. For instance, o_writer signals if the question is about a writer.

\section{B.2 Examples with multi_answers feature}

In the Logistic Regression analysis (Section 3.2), we create two features: multi_answers and multi_entities. Former captures the presence of multiple gold answers to the question in a given example, while latter signals presence of multiple person entities - all in either the answers, the question text or the document title for a given example. While multi_entities has consistent negative co-relation with model correctness (Appendix B), multi_answers has a disparate effect. Though it signals towards incorrectly answered examples in $\mathrm{NQ}$, it has a statistically significant positive correlation with model correctness for TriviaQA examples. Going through the examples, it reveals that TriviaQA uses multiple answers to give alternate formulations of an answer, which aids machine reading, while multiple NQ answers are often a sign of question ambiguity (Min et al., 2020).

To demonstrate that, we enlist here examples from development fold of both NQ (Appendix B.2.1) and TriviaQA (Appendix B.2.2) that have multiple gold answers.

\section{B.2.1 NQ examples with multiple answers:}

\section{id: $\mathbf{- 4 1 3 5 2 0 9 8 4 4 9 1 8 4 8 3 8 4 2}$}

Q: who carried the us flag in the 2014 olympics

A: Todd Lodwick

A: Julie Chu

id: $\overline{883871} 6539218945006$

$\mathrm{Q}:$ who says that which we call a rose

A: William Shakespeare

A: Juliet

id: -6197052503812142206

Q: who has won the most superbowls as a player

A: Charles Haley

A: Tom Brady

id: -2840415450119119129

Q: who started the guinness book of world records

A: Hugh Beaver

A: Norris and Ross McWhirter

id: 699742233861310118

Q: who played the nurse on andy griffith show

A: Langdon

id: -7064677612340044331

Q: who wrote the song if $i$ were a boy

Q: who wrote
A: BC Jean

A: BC Jean

A: Toby Gad 3248410603422198181

id: 3248410603422198181
Q: who conducted the opening concert at carnegie hall 


\begin{tabular}{|c|c|c|c|c|c|c|}
\hline Dataset & Features & Coef & SE & Wald (IWI) & $\mathbb{P}_{Z \sim \mathcal{N}}($ & \\
\hline \multirow{10}{*}{ NQ } & bias & +1.964 & 0.727 & 2.703 & 0.0069 & 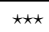 \\
\hline & multi_answers & -1.893 & 0.438 & 4.327 & 0.0000 & 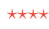 \\
\hline & o_not_found & -1.112 & 0.514 & 2.163 & 0.0305 & $\star \star$ \\
\hline & t_who & +0.773 & 0.280 & 2.764 & 0.0057 & $\star \star \star \star$ \\
\hline & o_law/crime & +0.729 & 0.738 & 0.989 & 0.3227 & \\
\hline & o_science/tech & -0.715 & 0.390 & 1.832 & 0.0670 & $\star$ \\
\hline & t_where & -0.695 & 0.645 & 1.078 & 0.2811 & \\
\hline & multi_entities & -0.678 & 0.342 & 1.979 & 0.0478 & $\star \star$ \\
\hline & t_what & +0.505 & 0.415 & 1.216 & 0.2240 & \\
\hline & o_stage & -0.444 & 0.607 & 0.732 & 0.4640 & \\
\hline \multirow{2}{*}{ Model Fit: } & g_female & +0.427 & 0.489 & 0.873 & 0.3829 & \\
\hline & o_executive & +0.425 & 0.684 & 0.622 & 0.5341 & \\
\hline \multirow{15}{*}{$78.09 \%$} & q_sim & +0.406 & 0.210 & 1.934 & 0.0531 & $\star$ \\
\hline & e_train_count & +0.353 & 0.178 & 1.979 & 0.0479 & $\star \star$ \\
\hline & o_others & +0.346 & 0.443 & 0.781 & 0.4346 & \\
\hline & o_music & +0.310 & 0.259 & 1.200 & 0.2302 & \\
\hline & o_fictional & -0.256 & 0.445 & 0.576 & 0.5644 & \\
\hline & o_politics & -0.197 & 0.304 & 0.646 & 0.5185 & \\
\hline & o_film/tv & +0.142 & 0.226 & 0.629 & 0.5294 & \\
\hline & t_when & -0.134 & 0.378 & 0.354 & 0.7233 & \\
\hline & g_male & -0.126 & 0.508 & 0.249 & 0.8033 & \\
\hline & e_train_count & +1.922 & 0.269 & 7.144 & 0.0000 & 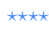 \\
\hline & bias & -1.024 & 0.291 & 3.516 & 0.0004 & 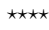 \\
\hline & o_film/tv & -0.910 & 0.470 & 1.934 & 0.0531 & $\star$ \\
\hline & multi_entities & -0.870 & 0.165 & 5.287 & 0.0000 & 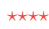 \\
\hline & t_what & -0.734 & 0.457 & 1.605 & 0.1085 & \\
\hline & o_translation & +0.671 & 1.439 & 0.466 & 0.6412 & \\
\hline \multirow[t]{3}{*}{ QB } & o_science/tech & -0.667 & 0.265 & 2.522 & 0.0117 & $\star \star$ \\
\hline & o_religion & -0.655 & 0.362 & 1.812 & 0.0700 & $\star$ \\
\hline & t_where & +0.545 & 0.615 & 0.885 & 0.3760 & \\
\hline \multirow{2}{*}{ Model Fit: } & o_history & -0.484 & 0.436 & 1.109 & 0.2673 & \\
\hline & o_writing & +0.402 & 0.189 & 2.128 & 0.0334 & $\star \star$ \\
\hline \multirow[t]{15}{*}{$71.90 \%$} & o_not_found & -0.382 & 0.349 & 1.093 & 0.2744 & \\
\hline & o_fictional & -0.368 & 0.540 & 0.681 & 0.4957 & \\
\hline & t_who & +0.363 & 0.183 & 1.990 & 0.0466 & $\star \star$ \\
\hline & o_artist & +0.194 & 0.268 & 0.721 & 0.4709 & \\
\hline & o_music & +0.123 & 0.249 & 0.492 & 0.6225 & \\
\hline & o_politics & -0.113 & 0.202 & 0.560 & 0.5756 & \\
\hline & o_philosophy & -0.082 & 0.259 & 0.315 & 0.7530 & \\
\hline & bias & -1.066 & 0.114 & 9.353 & 0.0000 & 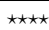 \\
\hline & o_education & -0.665 & 0.534 & 1.245 & 0.2132 & \\
\hline & o_economics & +0.503 & 0.579 & 0.869 & 0.3848 & \\
\hline & o_philosophy & -0.469 & 0.342 & 1.370 & 0.1706 & \\
\hline & o_religion & -0.443 & 0.255 & 1.738 & 0.0822 & * \\
\hline & -_law/crime & +0.412 & 0.218 & 1.890 & 0.0588 & $\star$ \\
\hline & multi_answers & +0.341 & 0.024 & 14.090 & 0.0000 & 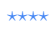 \\
\hline & t_who & +0.230 & 0.129 & 1.778 & 0.0754 & $\star$ \\
\hline \multirow{3}{*}{ TriviaQA } & o_science/tech & +0.219 & 0.151 & 1.452 & 0.1466 & \\
\hline & o_stage & -0.212 & 0.257 & 0.824 & 0.4098 & \\
\hline & o_politics & -0.208 & 0.095 & 2.177 & 0.0295 & $\star \star$ \\
\hline \multirow{2}{*}{ Model Fit: } & o_writing & +0.192 & 0.098 & 1.955 & 0.0506 & $\star$ \\
\hline & o_sports & -0.183 & 0.117 & 1.570 & 0.1164 & \\
\hline \multirow[t]{9}{*}{$60.18 \%$} & o_film/tv & +0.150 & 0.092 & 1.626 & 0.1039 & \\
\hline & t_when & -0.146 & 0.299 & 0.488 & 0.6258 & \\
\hline & o_not_found & -0.143 & 0.205 & 0.699 & 0.4845 & \\
\hline & o_executive & -0.130 & 0.207 & 0.625 & 0.5323 & \\
\hline & o_others & -0.101 & 0.152 & 0.665 & 0.5058 & \\
\hline & multi_entities & -0.099 & 0.077 & 1.290 & 0.1971 & \\
\hline & e_train_count & +0.081 & 0.075 & 1.079 & 0.2804 & \\
\hline & o_music & +0.022 & 0.097 & 0.223 & 0.8237 & \\
\hline & t_what & +0.018 & 0.134 & 0.132 & 0.8947 & \\
\hline
\end{tabular}

Table 5: Influential features revealed through Logistic Regression Analysis (Sec 3.2) over the demographic characteristics deemed significant through the $\chi^{2}$ test (Figure 1). We report the highly influential features with significance of $p$-value $<0.1$, both positive (blue) and negative (red), and bold the highly significant ones ( $p$-value $<$ $0.05)$. Number of $\star$ in the last column represents the significance level of that feature.

A: Walter Damrosch

A: Pyotr Ilyich Tchaikovsky

id: -3772952199709196386

$\mathrm{Q}:$ who founded amazon where is the headquarters of amazon

A: founded by Jeff Bezos

A: $\frac{\text { based in Seattle, Washington }}{4053461415821443645}$

Q: who wrote song what a friend we have in jesu

A: Joseph M. Scriven

A: Charles Crozat Converse

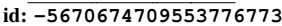

Q: who sings the theme song for the proud family

A: Solange Knowles

A: Solange Knowles

id: 2978779480736570480
Q: days of our lives cast doug and julie

A: Bill Hayes

A: Susan Seaforth

id: 6173192803639008655

Q: who has appeared in the most royal rumble

A: Isaac Yankem / " Diesel " / Kane

A: Shawn Michaels

id: $\mathbf{7 5 6 1 3 8 9 8 9 2 5 0 4 7 7 5 7 7 3}$

Q: who wrote the song stop the world and let me off

A: Carl Belew

A: W.S. Stevenson

id: -8366545547296627039

Q: who wrote the song photograph by ringo starr

A: Ringo Starr
A: George Harrison

id: -5674327280636928690

Q: who sings you're welcome in moana credits

A: Lin - Manuel Miranda

A: Jordan Fisher

id: -2432292250757146771

Q: who wrote the song $i$ hate you i love you

A: Garrett Nash

A: Olivia O'Brien

id: $\mathbf{- 3 6 3 2 9 7 4 7 0 0 7 9 5 1 3 7 1 4 8}$

$\mathrm{Q}:$ who is the owner of reading football club

A: Xiu Li Dai

A: Yongge Dai

id: 7163132803738849961

Q: who played guitar on my guitar gently weep

A: Eric Clapton

A: George Harrison

id: $\mathbf{1 3 1 8 0 3 1 8 4 1 8 1 3 1 2 1 3 8 7}$

Q: who sang the theme song to that 70 s show

A: Todd Griffin

id: 1393634180793653648

Q: who came up with the initial concept of protons and neutrons

A: Werner Heisenberg

id: 9134704289334516617

Q: who missed the plane the day the music died

A: Waylon Jennings

A: Tommy Allsup 
id: 8466196474705624263

Q: who was running as vice president in 1984

A: Congresswoman Ferraro

A: Vice President George H.W. Bush

id: $\mathbf{5 5 7 9 0 1 3 8 7 3 3 8 7 5 9 8 7 2 0}$

Q: who has won the canada open women's doubles

A: Mayu Matsumoto

A: Wakana Nagahar

id: 5584540254904933863

Q: who sang what are we doing in love

A: Dottie West

A: Kenny Rogers

id: -8677459248394445003
Q: who is hosting e live from the red carpet

A: Ryan Seacrest

A: Giuliana Rancic

id: $\overline{-1342189058950802702}$

Q: who made the poppies at tower of london

A: Paul Cummins

id: $\overline{6014950976264156000}$

Q: who sang never gonna let you go

A: Joe Pizzulo

id: -8052136860650205450

$\mathrm{Q}$ : who wrote the song rainy days and mondays

A: Roger Nichols

A: Paul Williams

id: 7903911150166287814

Q: what position did doug peterson play in the $n f l$

A: quarterback

A: holder on placekicks

id: $\overline{583026970021621830}$

Q: who invented the first home video security system

A: Marie Van Brittan Brown

A: Marie Van Brittan Brown

id: 5427679691711111925

Q: who were the two mathematicians that invented calculus

A: Isaac Newton

A: Gottfried Leibniz

id: $\overline{-9163844183450408581}$

Q: nba record for most double doubles in a season

A: Tim Duncan leads the National Basketball Association (NBA) in the points - rebounds

combination with 840

A: John Stockton leads the points - assists combination with 714

id: $\mathbf{- 8 1 0 9 3 6 7 5 3 7 6 9 0 3 4 3 8 9 5}$

Q: who were the twins that played for kentucky

A: Andrew Michael Harrison

A: Aaron Harrison

id: $\overline{4784420206031467202}$

Q: who wrote he ain't heavy he's my brother lyrics

A: Bobby Scott

id: 4136958282795887427

Q: who opens the church of the holy sepulchre

A: the Nusaybah family

A: the Joudeh Al - Goudia family

id: $\mathbf{- 2 6 1 0 2 0 9 5 6 0 6 9 9 5 2 8 8 9 6}$

Q: who is the writer of 50 shades of grey

A: Erika Mitchell Leonard

A: E.L. James

id: $\overline{8968036245733884389}$

Q: when did stephen curry won the mvp award

A: 2015 ,

id: -1899514742808499173

Q: who are nominated for president of india 2017

A: Ram Nath Kovind

A: Meira Kumar

$-3019484115332998709$

Q: what movie is count on me by bruno mars in

A: A Turtle's Tale : Sammy's Adventures

A: Diary of a Wimpy Kid : The Long Haul

id: 810060125994185205

Q: who was the first to say i'm going to disney world

A: Dick Rutan

id: 339027965927992295

Q: who sings the whiskey ain't workin anymore

Q: who sings

A: Travis Trit

id: Marty Stuart

id: 5995814638252489040

A: Peter Hansen

id: 3723628014502752965

Q: who wrote cant get you out of my head lyrics

A: Cathy Dennis

A: Rob Davis

id: $\overline{3886074} 985605209321$

Q: who sings find out who your friends are with tracy lawrence

A: Tim McGraw

A: Kenny Chesney

id: $\mathbf{3 6 2 4 2 6 6 5 1 8 3 2 8 7 2 7 0 4 0}$

Q: who invented the printing press and what year

A: Johannes Gutenberg

A: Johannes 6

id: $\frac{-4951004239400083779}{-49}$

Q: who plays chris grandy in 13 going on 30
A: Jim Gaffigan

id: $\overline{\mathbf{2 6 7 2 7 2 1 7 4 3 9 1 1 1 1 7 1 8 5}}$

Q: who developed a set of postulates to prove that specific microorganisms cause disease

A: Robert Koch

A: Friedrich Loeffler

id: $\mathbf{2 1 6 6 0 9 2 8 0 1 7 9 7 5 1 5 5 0 0}$

Q: who is the director of taarak mehta ka ooltah chashmah

A: Harshad Joshi

A: Malav Suresh Rajd

id: -3389723371168293793

Q: who has the most olympic medals in figure skating

A: Tessa Virtue

id: -8391680223788694572

Q: who wrote if $i$ were a boy reba or beyonce

A: BC Jean

A: Toby Gad

id: $\overline{1070572237499172286}$

Q: who wrote the song after you've gone

A: Turner Layton

A: Henry Creame

id: 2343902375984110832

Q: who does the voice of mickey mouse on mickey mouse clubhouse

A: Wayne Allwine

A: Bret Iwan

id: $\mathbf{7 0 1 3 8 6 3 9 3 9 8 0 3 4 9 5 6 9 4}$

Q: who sings love me tender in princess diaries 2

A: Norah Jones

id: 4925057086725798331

Q: who wrote yakkity yak don't talk back

A: Jerry Leiber

A: Mike Stolle

id: 647605647914971565

Q: who wrote lyrics for phantom of the opera

A: Charles Hart

A: Richard Stilgoe

id: -6371603500131574271

Q: who sings somebody's watching me with michael jackson

A: Rockwell

A: Jermaine Jackson

id: -4036503601399675973

Q: when did michael jordan return to the nba

A: 1995

id: $\overline{4323871331649279373}$

Q: who invented the printing press and in what year

A: Johannes Gutenberg

A: 1440

id: $\mathbf{7 2 3 4 2 7 7 1 2 3 6 4 6 8 5 2 4 4 7}$

Q: who sings war don't let me down

A: American production duo The Chainsmokers

A: vocals of American singer Daya

id: $\mathbf{4 2 4 5 7 9 8 0 6 6 9 2 3 2 2 3 4 5 7}$

Q: who has the most all star mvp awards

A: Bob Pettit

A: Kobe Bryant

id: -3585157729928173881

Q: who plays hulk in the thor and avengers series of movies

A: Fred Tatasciore

id: -7892904540301629325

Q: who wrote the song going to kansas city

A: Jerry Leiber

id: $\overline{1838851770314085590}$

Q: who plays sheila carter on the bold and the beautiful

A: Kimberlin Brown

A: Michelle Stafford

\section{B.2.2 TriviaQA multi-answer examples:}

We randomly sample 100 examples from TriviaQA where questions had multiple answers.

id: sfq_6110

Q: On which island in the North Sea did both St Aidan and St Cuthbert live?

A: Lindisfarne

A: $\overline{\text { LINDISFARNE }}$

id: tc_1008

Q: To the nearest two, how many tennis Grand Slam titles did Jimmy Connors win?

A: 10

A: $\overline{\operatorname{ten}}$

id: $\overline{\text { sfq } \_26211}$

Q: In the TV series Doctor Who, who was the creator of the Daleks and arch enemy of the

Doctor?

A: Davro

A: $\overline{\text { Creator of the Daleks }}$

id: $\mathbf{s f q} 22212$

Q: In which book of the bible is the story of Samson and Delilah?

A: Judge (disambiguation)

A: Judges

id: bt 1538

$\mathrm{Q}$ : What is cartoon character Mr. Magoo's first name

A: Quincy (disambiguation)

A: Quincy 
id: $q z \_2444$

Q: What is Robin Williams character called in Good Morning Vietnam?

A: Adrian

Q: What was the first name of the jazz trombonist Kid Ory?

A: Eadweard

id: qw 1606

id: qw_1606
Q: Which of Queen Elizabeth's children is the lowest in succession to (i.e. furthest away

from) the throne?

A: Anne

A: $\overline{\text { Ann (name) }}$

id: odq1_5503

Q: "Which radio comedian's catchphrase was "'daft as a brush"'?"

A: KEN PLATT

A: Ken Platt

id: qg_2992

Q: According to Sammy Haggar, what can't he drive?

A: 55

A: fifty-five

id: qf_3440
Q: What was Grace Darling's father's job?

Q: What was Grace Darin
A: Lighthouse-keeper

A: Lighthouse keeper

id: qw_12369

Q: "What year did Jean-Francois Champollion publish the first correct translation of Egyp-

tian hieroglyphs from the Rosetta Stone, the Roman Catholic Church take Galileo Galilei's

"'Dialogue"' off their list of banned books, and Britain repeal the death penalty for over 100 crimes?"

A: one thousand, eight hundred and twenty-two

A: 1822

id: qw_ 4143

Q: What is the title of the most famous painting by Franz Hals?

A: Laughing Cavalier

A: The Laughing Cavalier

id: qb_2647

Q: What is the title of the 1944 film starring Barbara Stanwyck as the wife who seduces an

insurance salesman into killing her husband?

A: Double indemnity (disambiguation)

A: Double Indemnity

Q: Who was the choreographer of the dance troupe Hot Gossip?

A: Arlene Philips

A: Arlene Phillips

id: tc_719

Q: River Phoenix died during the making of which movie?

A: Dark Blood (film)

A: Dark Blood

id: $\overline{\text { sfq_19457 }}$

Q: Who won the first ever boxing gold for women? She shares her surname with two US

Presidents.

A: Nicola Adams

id: sfq 8996

Q: Actor Norman Painting died in November 2009, which part in a log running radio series

did he make his own?

A: PHIL ARCHER

A: Phil Archer

id: $\operatorname{dpgl} 6111$

Q: Which Jersey-born actor played Superman in Man of Steel?

A: Henry Cavill

A: Henry William Dalgliesh Cavill

id: $q z \_2135$

Q: Name the game show, presented by Leslie Grantham and Melinda Messenger, where contestants were set physical and mental challenges?

A: Fort Boyard (disambiguation)

A: Fort Boyard

id: odq1_3205

Q: Who wrote the novel 'The Beach' on which the film was based?

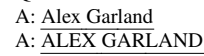

id: $q z 2999$

Q: In what year did Edward Vlll abdicate?

A: one thousand, nine hundred and thirty-six

A: 1936

id: tc_723

Q: Which artist David was born in Bradford UK?

A: Hockney

A: David Hockney

id: odq1_3708

Q: Three Liverpool players were in the 1966 England World Cup winning squad. Roger

Hunt and Ian Callaghan were two - who was the third?

Hunt and lan Callas

A: Gerry Byrne

id: qw_ 11151

Q: Which artist has a daughter and two sons with Jane Asher, whom he married in 1981?

A: Gerald Anthony Scarfe

A: Gerald Scarfe

id: qb_3652

$\mathrm{Q}:$ Who wrote the novel 'The Eagle Has landed'?

A: Harry Patterson

id: odgl 14683

Q: Who presents the BBC quiz show 'Perfection'?

Q: Who presents

A: Nick Knowles

id: $\mathbf{s f q} \mathbf{9 4 6 4}$

id: $\mathbf{s f q} 9464$
Q: Who succeeded Brian Epstein as manager of The Beatles?
A: Allan Klein

A: $\overline{\text { Allen Klein }}$

id: wh_2615

Q: In which year did both T-Rex's Marc Bolan and Elvis Presley die?

A: 1977

A: one thousand, nine hundred and seventy-seven

id: sfq_9018

Q: Who played Hotlips Houlihan in the 1972 film MASH?

A: Sally Kellerman

A: SALLY KELLERMAN

id: qz_1516

A: Ken Bates

A: Kenneth Bates

id: sfq_23289

Q: What was the middle name of the author William Thackeray?

A: Makepeace

A: MAKEPEACE

id: sfq 7589

Q: What was the name of the older brother of Henry 8th?

A: Arthur

A: $\overline{\text { Arthur (name) }}$

id: odq1_10316

Q: Which actor played 'Hadley', in the TV series of the same name?

A: GERALD HARPER

A: Gerald Harper

id: sfq_12933

Q: Operation Barbarossa, Hitler invades Russia.

A: one thousand, nine hundred and forty-one

A: 1941

id: $\mathbf{q w}$-5050
Q: "Which Italian nobel prize winner (1934) wrote novels such as "'Mal Gioconda"" and

Q: "Which Italian nobel prize winner
switched to writing plays in 1910?"

switched to writing
A: Pirandello

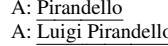

id: bt_2403

Q: What was the name of the driver of the mail train robbed by the great train robbers

A: Jack Mills (train driver)

A: Jack Mills

id: sfq_2189

Q: What was the name of the private eye played by Trevor Eve on TV in the '70s?

A: Shoestring (TV series)

A: Eddie Shoestring

id: qb_5431

Q: Brazilian football legend Pele wore which number on his shirt?

A: 10

A: $\frac{10}{\operatorname{ten}}$

id: $\overline{q b} \_4726$

Q: Michael J Fox travels back to which year in the Wild West in the 1990 film 'Back To The

Future Part III'?

A: one thousand, eight hundred and eighty-five

A: 1885

id: odq1_8275

Q: Later a 'Blue Peter' presenter, who played 'Steven Taylor', an assistant to William Hart-

nell's 'Doctor Who'?

A: PETER PURVES

A: Peter Purves

id: sfe 962

id: sfq_962

A: Salford

A: Salford (disambiguation)

id: $t c \_1348$

Q: In the late 60s Owen Finlay MacLaren pioneered what useful item for parents of small chldren?

A: Baby Buggy

A: Baby buggy

id: qw_12732

Q: General Franco, the Spanish military general, was head of state of Spain from October

1936 following the Spanish Civil War, until when?

A: 1975

A: one thousand, nine hundred and seventy-five

id: $\mathrm{bt} \_4495$

Q: Which of the Great Train Robbers became a florist outside Waterloo station until he was

found hanged in a lock up

A: Buster Edwards

A: Ronald \%22Buster\%22 Edwards

id: sfq_20394

Q: Which TV presenter, who died in February 2013, was for over 20 years the host of ' $M r$ \& Mrs'?

A: Derek Batey

A: Derek Beatty

id: odq1_12918

id: odq1_12918

A: Tim Farron

A: Timothy Farron

id: odq1_13785

Q: Who wrote the lyrics for 'Sing', written to celebrate the Queen's Diamond Jubilee?

A: Gary Barlow

A: GARY BARLOW

id: sfq 20830

Q: Which top National Hunt trainer's establishment is based at Seven Barrows?

A: NICKY HENDERSON

A: Nicky Henderson

id: wh 2133

Q: Which T.V. Quiz show host used the catchphrase :- If its' up there, I'll give you the money myself?

A: LES DENNIS

A: $\underline{\text { Les Dennis }}$ 


\section{id: sfq_15663}

Q: The 27 episodes of which sitcom featuring Julia Mckenzie, Anton Rodgers and Ballard Berkley were first broadcast in the 1980s?

A: Fresh Fields (TV series)

A: Fresh Fields

Q: odq1_4871

Q: When $U$ : $P$

A: WAITING FOR A TRAIN

A: Waiting for a Train

id: bb_6592

Q: Which artist was born in Bradford in 1937 ?

A: Hockney

A: David Hockney

id: qw_10270

Q: Argentina invaded UK's Falkland Islands, Israel invaded Southern Lebanon, Canada became officially independent of the UK, Leonid Brezhnev, leader of the USSR, died, all in what year?

A: one thousand, nine hundred and eighty-two

A: $\frac{1982}{198}$

\section{id: $\overline{b t} 4206$}

Q: Who was the first woman to be seen on Channel 4

A: Carol Vorderman

A: Carol Voderman

id: qw_8871

Q: Lieutenant General James Thomas Brudenell, who commanded the Light Brigade of the British Army during the Crimean War, was the 7th Earl of what?

A: Cardigan

A: Cardigan (disambiguation)

id: sfq 13639

Q: Which model village did Samuel Greg build to house workers at his nearby Quarry Bank Mill?

A: Styal

id: $\overline{\text { tc_812 }}$

Q: Who was the defending champion when Martina Navratilova first won Wimbledon sinles?

A: Virginia Wade

A: Sarah Virginia Wade

id: $\mathbf{s f q} 11790$

Q: Opened in 1963, which London nightclub did Mark Birley name after his then wife?

A: Annabel's

A: ANNABELS

id: qw_8397

Q: In 1995, Steffi Graf became the only tennis player to have won each of the four grand

Q: In 1995, Steff Graf becane
slam events how many times?

A: four

d: $\mathbf{d p q} 13151$

Q: On which river does Ipswich stand?

A: Orwell (disambiguation)

A: Orwell

\section{id: qw_14634}

Q: "Which Bob Dylan song begins "'You got a lotta nerveTo say you are my friend. When I was down, You just stood there grinning""?"

A: Positively Fourth Street

A: Positively 4th Street

id: dpq1_1801

Q: Nick Begs was lead singer with which 80 's pop band?

A: Kaja Googoo

A: $\underline{\text { Kajagoogoo }}$

id: qw_16011

Q: In 1483, who was appointed the first grand inquisitor of the Spanish Inquisition?

A: Torquemada (disambiguation)

A: Torquemada

id: qw_1933

Q: What remake of a British science-fiction serial broadcast by BBC Television in the summer of 1953 was staged live by BBC Four in 2005 with actors Jason Flemyng, Mark Gatiss, Andrew Tiernan, Indira Varma, David Tennant and Adrian Bower?

A: Quatermass experiment

A: Quatermass experiment

id: odq1 2323

id: odq1_2323 Which 2009 film is a biopic of John Lennon?

Q: Which 2009 film is a

A: 'NOWHERE BOY'

A: Nowhere Boy

Q: 'The Battle of Trafalgar' is the work of which British painter?

A: Joseph Turner

A: Joseph Turner (disambiguation)

id: qw 4463

$\mathrm{Q}$ : Who discovered the two moons of Mars in 1877?

A: Asaph Hall

A: Asaph Hall III

A: Asaph Hall

id: qz_1111 2 Which brand of beer does Homer Simpson drink regularly?

A: Duff

A: Duff (disambiguation)

id: wh_8

Q: In the novel 'Treasure Island' name the pirate shot dead by Jim Hawkins in the rigging of the Hispaniola

A: Israel Hands

A: ISRAEL HANDS

id: qg_3884

Q: Dow Constantine and Susan Hutchinson are currently running for was position?

A: King County executive

id: $\mathbf{s f q} 25940$

Q: Which actor played the part of Ross Poldark in the BBC's mid 1970's television series?
A: Robin Ellis

A: $\overline{\text { ROBIN ELLIS }}$

id: odq1_12777

Q: For which 1960 film did Billy Wilder become the first person to win three Oscars for the same film?

A: The Apartment

A: The apartment

Q: Famous for 'Die Welt als Wille und Vorstellung', Arthur Schopenhauer (1788-1860) was

a German?

A: Philosophers

A: Philosopher

id: qb_8589

Q: What is the nickname of the frontiersman Nathaniel Poe, played by Daniel Day Lewis, in the 1992, film 'The Last of the Mohicans'?

A: Hawkeye

A: Hawkeye (disambiguation)

id: bt_2852

Q: Who played the part of Tina Seabrook in Casualty

A: Claire Woodrow

A: Claire Goose

id: wh 557

Q: Who duetted with Syd Owen on the single Better Believe It, which was released as part

of the Children in Need appeal in 1995 ?

A: PATSY PALMER

A: Patsy Palmer

id: odq1_3979

Q: What is the name of the character played by Nicole Kidman in the film 'Moulin Rouge'?

A: Satine

A: 'SATINE'

id: odq1_10365

Q: Which British girl won the Women's Junior Singles title at Wimbledon this year (2008)?

A: LAURA ROBSON

A: Laura Robson

id: qf_173

Q: In what year did Elvis Presley and his parents move from Tupelo to Memphis?

A: one thousand, nine hundred and forty-eight

A: 1948

id: tc_1468

Q: What was Pete Sampras seeded when he won his first US Open?

A: twelve

A: 12

id: qf_2679

Q: Who on TV has played a scarecrow and a Time Lord?

A: John Pertwee

A: Jon Pertwee

id: $\mathbf{s f q} \mathbf{1 1 8 4 4}$
Q: In which year was Olaf Palme assassinated and the Chernobyl nuclear power station

exploded?

A: 1986

A: one thousand, nine hundred and eighty-six

id: $\mathrm{qf} \_3578$

Q: Cassandra was the pseudonym of which writer in the Daily Mirror?

A: William Neil Connor

A: William Connor

id: $q z \_3898$

Q: How many times did Steffi Graf win the Ladies Singles at Wimbledon?

A: seven

id: qw 6782

Q: What is the disease that Stephen Hawking has?

A: Motor neuron disease

A: $\overline{\text { Motor neuron diseases }}$

id: qw_15255

Q: "How many films were made by director Sir Peter Jackson from Tolkien's short book, "'The Hobbit"'?"

A: 3

A: three

id: $\overline{\text { sfq_ }} 7168$

Q: Who invented the wind-up radio?

Q: Who invented
A: Trevor Bayliss

A: TREVOR BAYLISS

A: TREVOR BAY

id: sfq_14433
Q: In Pride and Prejudice what was the first name of Mr Darcy?

A: Fitzwilliam (disambiguation)

A: Fitzwilliam

id: odq1_8230

Q: Which single by 'Leapy Lee' reached number two in the UK charts in 1968 ?

A: 'LITTLE ARROWS'

A: Little Arrows

id: dpql 1416

$\mathrm{Q}:$ Whose is the first tale in Chaucer's Canterbury Tales?

A: The Knight

A: Knight (disambiguation)

id: qw_1672

Q: Which womens squash player won the World Open four times (1985, 1987, 1990 \& 1992)

and the British Open eight times?

A: Susan Devoy

A: Susan Elizabeth Anne Devoy

id: $q z \_832$

Q: Who wrote the novels About A Boy, How To Be Good and High Fidelity?

A: Nick Hornby

A: $\overline{\text { Hornby, Nick }}$

id: sfq_22884

id: $\mathbf{s f q} \mathbf{2 2 8 8 4}$ Q: Which TV series was about a pop group called 'Little Ladies' featuring Charlotte Corn-

well, Julie Covington and Rula Lenska?

A: Rock Follies

A: Rock Follies of ' 77 
id: odq1_10746

Q: Who wrote the 1951 novel 'The Caine Mutiny'?

A: HERMAN WOUK

A: Herman Wouk

id: $\mathrm{bb} \_285$

Q: Said to refer erroneously to the temperature at which book paper catches fire, the title

of Ray Bradbury's 1953 novel about a futuristic society in which reading books is illegal, is

called 'Fahrenheit...' what? 972; 451; 100; or 25?

A: 451

A: four hundred and fifty-one

id: odql 7290

Q: Who was the driver of the limousine at the time of Diana Princess of Wales' death?

A: HENRI PAUL

A: Henri Paul

id: $\mathbf{s f q} \mathbf{4 3 6 8}$
Q: Which island in the Grenadines of St. Vincent was bought by Colin Tennant in 1958?

Princess Margaret built a holiday home there in the 1960's.

A: MUSTIQUE

A: $\frac{\text { Mustique }}{\text { idgl } 5476}$

Q: Which pop star had the real name of Ernest Evans?

A: Chubby Checker

A: 'CHUBBY CHECKER'

id: tc_980

Q: "Which supermodel said, "'I look very scary in the mornings?"

A: We don't wake up for less than $\$ 10,000$ a day

A: Linda Evangelista 